\title{
Evaluación nutricional de tres especies de árboles forrajeros: Morus alba, Gliricidia sepium y Trichanthera gigantea en bovinos fistulados
}

\section{Nutritional evaluation of three species of fodder trees: Morus alba, Gliricidia sepium and Trichanthera gigantea in fistulated cattle}

\author{
Niño Guayacán Mauricio ${ }^{1}$ y Roa Vega María Ligia² \\ ${ }^{1} \mathrm{MVZ}$. Universidad de los Llanos y \\ 2Z. MSc Docente Universidad de los Llanos \\ mroa@unillanos.eu.co
}

Recibido 12 de Diciembre 2013, Aceptado 26 de Septiembre 2014

\section{RESUMEN}

Este trabajo se llevó a cabo en la granja y en el laboratorio de Nutrición y Alimentación animal de la Universidad de los Llanos, localizada en Villavicencio (Meta), se utilizaron tres novillas Pardo Suizo-Criolla, fistuladas en rumen, las cuales fueron alimentadas con Brachiaria decumbens, sal mineralizada y agua a voluntad, además se suplementaron con $2 \mathrm{~kg}$ de materia seca de tres especies de forrajes arbóreos conformando tres tratamientos así: T1 = Morus alba, T2 = Trichanthera gigantea y T3 = Gliricidia sepium, para garantizar su consumo se adicionaron $300 \mathrm{gr}$ de melaza en el momento del suministro. Los forrajes fueron evaluados en el laboratorio mediante análisis proximal y determinación de la fibra detergente neutro (FDN) y fibra detergente ácido (FDA). En campo se desarrollaron tres periodos experimentales aplicando un diseño estadístico cuadrado latino $3 \times 3$ con el objetivo de determinar la degradabilidad ruminal y tasa de degradación, utilizando la técnica de las bolsas de nylon, en las cuales se colocaron $5 \mathrm{gr}$ de muestra de cada tratamiento en diferentes horas $(6,12,24,48 \mathrm{y}$ 72). Los nutrientes evaluados fueron: materia seca (MS), FDN, FDA y nitrógeno total (NT). En el líquido ruminal se realizaron mediciones de $\mathrm{pH}$ y nitrógeno amoniacal $\left(\mathrm{N}^{\left.-\mathrm{NH}_{3}\right)}\right.$ a las $0,4,8$ y 12 horas de incubación. Los resultados del análisis bromatológico indicaron que Gliricidia sepium contiene mayor cantidad de proteína (22.1\%) en comparación con Morus alba y Trichanthera gigantea (18.6 y 
19.2\% respectivamente). Morus alba únicamente contiene $28.3 \%$ de FDN con relación a las otras dos especies cuyo valor supera el 40\%, así mismo presentó las mayores tasas de degradación en cuanto MS, NT, FDA y FDN $(P<0.05)$, alcanzando este último nutriente la mayor degradabilidad a las 72 horas (81.9\%). Aunque Gliricidia sepium obtuvo una tasa de degradación rápida de la FDN (9.4\% material degradado/hora) no se mantuvo igual esta tendencia hasta las 72 horas, siendo la degradabilidad de la FDN más baja de las tres especies estudiadas $(\mathrm{P}<0.05)$. Trichanthera gigantea presentó la tasa de degradación más baja en lo que corresponde a la MS, FDN, FDA y NT $(P<0.05)$, este último nutriente tiene una degradación lenta en un comienzo, lo que puede estar influenciado por la dificultad en la liberación de nitrógeno por la lenta degradación de la pared celular, sin embargo la degradabilidad del NT a las 72 horas es mayor que la de Gliricidia sepium. Los resultados de la tasa de degradación de la materia seca en Trichanthera gigantea y Morus alba son menores a los reportados en la literatura. Morus alba mostró la degradabilidad de nitrógeno total más elevada en todas las horas $(P<0.05)$. Los parámetros evaluados en líquido ruminal en lo que se refiere a nitrógeno amoniacal y $\mathrm{pH}$ no se observaron diferencias en las tres especies estudiadas. Se concluye que la mayor degradabilidad ruminal a las 72 horas de la MS, FDN, FDA y NT la presentó Morus alba en comparación con Trichanthera gigantea y Gliricidia sepium.

Palabras clave: Morus alba, Trichanthera gigantea, Gliricidia sepium, degradabilidad ruminal.

\section{ABSTRACT}

This experiment was carried out on the farm and in the laboratory of Animal Nutrition and Food of the University of the Llanos, located in Villavicencio (Meta), three heifers Brown Swiss-Creole, fistulated rumen were used, which were fed Brachiaria decumbens, mineralized salt and water ad libitum also supplemented with $2 \mathrm{~kg}$ dry matter of three species of tree fodder thus forming three treatments: $\mathrm{T} 1$ = Morus alba, $\mathrm{T} 2$ = Trichanthera gigantea and T3 = Gliricidia sepium, to ensure consumption $300 \mathrm{gr}$ of molasses were added at the time of delivery. The forages 
were evaluated in the laboratory by proximal analysis and determination of neutral detergent fiber (NDF) and acid detergent fiber (ADF). Field three experimental periods using a Latin square experimental design $3 \times 3$ in order to determine the ruminal degradability and degradation rate, using the technique of the nylon bags were developed, in which were placed $5 \mathrm{gr}$ of sample from each treatment at different times $(6,12,24,48$ and 72). Nutrients assessed were: dry matter (DM), $\mathrm{NDF}, \mathrm{ADF}$ and total nitrogen (TN). In ruminal $\mathrm{pH}$ measurements and liquid ammonia nitrogen $\left(\mathrm{N}-\mathrm{NH}_{3}\right)$ at $0,4,8$ and 12 hours were performed. The results of the compositional analysis indicated that Gliricidia sepium contains more protein (22.1\%) compared with Morus alba and Trichanthera gigantea (18.6 and 19.2\% respectively). Morus alba contains only $28.3 \%$ FDN in relation to the other two species whose value exceeds $40 \%$, also had the highest rates of degradation as DM, TN, ADF and NDF $(P<0.05)$, reaching the latter nutrient greater degradability after 72 hours (81.9\%). Although Gliricidia sepium obtained a rapid degradation rate of FDN (9.4\% degraded material/hour) not equal this trend continued up to 72 hours, with the degradability of the lowest FDN of the three species studied $(P<0.05)$. Trichanthera gigantea presented the lowest rate of degradation which corresponds to DM, NDF, ADF and TN $(P<0.05)$, the latter nutrient has a slow degradation in the beginning, which can be influenced by the difficulty in releasing nitrogen by the slow degradation of the cell wall, however the degradability of NT at 72 hours is greater than Gliricidia sepium. The results of the degradation rate of dry matter in Trichanthera gigantea and Morus alba are lower than those reported in the literature. Morus alba degradability showed higher total nitrogen at all hours $(\mathrm{P}<0.05)$. The parameters evaluated in ruminal fluid as regards $\mathrm{pH}$ and ammonium nitrogen no differences were observed. We conclude that greater ruminal degradability within 72 hours of DM, NDF, ADF and TN was observed in Morus alba compared to Trichanthera gigantea and Gliricia sepium.

Keywords: Morus alba, Trichanthera gigantea, Gliricidia sepium, ruminal degradability. 


\section{INTRODUCCIÓN}

La presente investigación propone demostrar que los forrajes arbóreos en el Piedemonte Llanero son un buen recurso para ser utilizados en la alimentación de rumiantes, porque tienen varias ventajas tales como: un buen nivel de proteína y minerales, adicionalmente se pueden obtener otros beneficios dentro de las explotaciones ganaderas como es ayudar a evitar la erosión y a conservar el agua lo que favorece el mantenimiento de especies silvestres de la zona. Por lo tanto este trabajo tiene como objetivo profundizar en la investigación sobre la calidad nutricional de las siguientes tres especies forrajeras: Morera (Morus alba), Matarratón (Gliricidia sepium) y Nacedero (Trichanthera gigantea) en bovinos fistulados, con estos resultados se contribuye a lograr un desarrollo gradual en busca de una tecnificación de los sistemas sostenibles de producción pecuaria aprovechando así, las ventajas socioeconómicas y ambientales que ofrece esta zona.

Para realizar la degradación de los forrajes el rumen cuenta con diversos microorganismos como bacterias, hongos y protozoos, que son los encargados de romper las partículas para facilitar el aprovechamiento de los nutrientes que finalmente va a utilizar el animal en sus procesos productivos, además este proceso digestivo se encuentra influenciado por varios factores, los cuales son principalmente: edad del animal, tipo de producción, calidad y cantidad de la fibra del forraje, nivel nutricional del animal, frecuencia de alimentación y uso de suplementos (Roa, 1992).

Es así, que la digestión de la proteína en el rumen depende de su solubilidad para que sea atacada por las bacterias proteolíticas las cuales requieren para su crecimiento un $\mathrm{pH}$ cercano a la neutralidad, de otra parte el proceso se ve afectado por la concentración de nitrógeno amoniacal $\left(\mathrm{N}_{-} \mathrm{NH}_{3}\right)$, si estos niveles son altos las bacterias no degradan la proteína, por lo tanto esta será digerida por enzimas producidas en el estómago y en el intestino delgado, si las cantidades de $\mathrm{N}-\mathrm{NH}_{3}$ son bajas en el rumen, las bacterias utilizarán la proteína del alimento para 
la obtención de nitrógeno, incrementando la degradación de este nutriente en el rumen (Orskov, 1988; Wattiaux y Howard, 2000).

La degradación de la fibra del forraje lo realizan bacterias que producen la celulasa y que pertenecen al género Ruminococcus Bacteroides y Butyrivibrio, en ésta digestión también intervienen factores que afectan la población de bacterias celulolíticas como son el tipo de dieta y forraje, tasa de pasaje, tamaño de partículas y otros. Además la fibra del alimento tiene como función mantener cercano a la neutralidad el $\mathrm{pH}$ del rumen lo que favorece el incremento de la población de las bacterias celulolíticas y por tanto la disponibilidad de ácidos grasos volátiles que son la principal fuente de energía del rumiante (Owens y Goetsch, 1988).

Se han establecido parámetros que evalúan el aprovechamiento de los nutrientes en el rumen, como es el caso de las tasas de degradación las cuales miden la cantidad de alimento degradado por unidad de tiempo, determinando que existe una fracción digestible de la pared celular y otra que es potencialmente digestible y está sujeta a las condiciones del rumen para que sea aprovechada parcialmente o en su totalidad (Wilkings, 1990; Frioni, 1999).

Flores et al., (1998) adelantaron una investigación con el objetivo de generar información sobre los parámetros de degradabilidad ruminal de la materia seca de ocho especies arbóreas y arbustivas tropicales entre las cuales se estudiaron la morera (Morus alba) y el nacedero (Trichanthera gigantea) para comparar su composición nutricional y además su aprovechamiento mediante la evaluación de la degradabilidad in situ en rumen utilizando la técnica de la bolsa de nylon. La digestibilidad de la materia seca fue mayor para morera, cayeno y nacedero, las cuales reportaron valores de 74,72 y $67 \%$ respectivamente, comparándolas con las demás especies evaluadas como la Calliandra calothyrsus, Cratylia argéntea, Erythrina berteroana y Leucaena leucocephala que obtuvieron degradaciones menores del $60 \%$. No hubo diferencias en la tasa de degradabilidad de la materia seca de las diferentes especies, siendo numéricamente más elevada la de morera $33.2 \%$ de material degradado/hora con relación a la de nacedero que fue de $6.3 \%$ 
de material degradado/hora. En cuanto al contenido de proteína, FDN y FDA los valores para nacedero y morera fueron los siguientes: $19.9,40.7$ y $33.9 \%$, para la primera $24.4,29.8$ y $18.8 \%$ para la segunda, respectivamente.

Las bondades nutricionales de las especies arbustivas forrajeras también han sido estudiadas en otras especies animales, en cabras Rodríguez y Elizondo, (2012) encontraron una digestibilidad de MS en $49.18 \%$, Proteína cruda $59.82 \%$, FDN $57.83 \%$ y FDA $55.30 \%$. De manera similar en conejos de engorde Nieves et al., (2006) encontraron en Morus alba una digestibilidad de la materia seca de $61.65 \pm$ $10.83 \%$, en la fibra detergente neutro $58.96 \pm 11.05 \%$ y fibra detergente acido $48.68 \pm 12.71 \%$. Por otro lado, respecto a Gliricidia sepium Hurtado et al., (2012) en cuyes determinaron una digestibilidad de la MS de $49.9 \%$, proteína bruta $91.6 \%$, y fibra bruta $75.0 \%$, mientras que Suárez et al., (2008) estimaron una digestibilidad in vitro de la MS de Trichantera gigantea en $44.46 \%$

\section{METODOLOGÍA}

La investigación se realizó en la granja y en el laboratorio de Nutrición y Alimentación animal de la Universidad de los Llanos, localizada en la ciudad de Villavicencio (Meta), a $12 \mathrm{~km}$ del casco urbano Vía a Puerto López, a una altitud de 465 m.s.n.m., una temperatura promedio de $27^{\circ} \mathrm{C}$, con un rango de precipitación anual entre 1900 y 2300 mm y una humedad relativa del $80 \%$.

Se emplearon tres novillas Pardo Suizo x Criolla, fistuladas a nivel del rumen, con una edad promedio de treinta meses, que fueron alimentadas en pastoreo con Brachiaria decumbens, sal mineralizada y agua a voluntad, además se suplementaron con tres especies de forrajes arbóreos para formar un cuadrado latino $3 \times 3$ con los siguientes tratamientos: T1 = $2 \mathrm{~kg}$ de Morera (Morus alba), T2 $=2 \mathrm{~kg}$ de nacedero (Trichanthera gigantea) y T3 $=2 \mathrm{~kg}$ de matarratón (Gliricidia sepium) todos en base seca, para garantizar el consumo de estas especies se mezclaron con $300 \mathrm{gr}$ de melaza. La cantidad de forraje suministrada se determinó haciendo pruebas de consumo previamente durante 10 días, en lo que se observó una buena aceptación de esa cantidad de los forrajes. Previamente al trabajo de 
campo se cortaron ramas tiernas (aproximadamente 4 meses de retoño) de las arbóreas mencionadas anteriormente, luego se secaron por separado en latas de zinc al aire libre, después esta materia seca se mezcló con melaza para suministrarla a los animales, con los cuales se desarrollaron tres periodos experimentales de 12 días cada uno, distribuidos así: los primeros 8 días fueron de adaptación a la dieta con árboles forrajeros y los otros 4 se tomaron las muestras para determinar su aprovechamiento en el rumen.

Las pruebas in situ en rumen se realizaron utilizando la técnica de las bolsas de nylon (Méhrez y Orskov, 1977), las cuales tenían un tamaño de $20 \times 10 \mathrm{~cm}$ con un poro promedio de 40 micras aproximadamente con el fin de evitar la salida de residuos de forraje, estas bolsas se secaron previamente a $60^{\circ} \mathrm{C}$ por 24 horas con el fin de llevarlas a peso constante y en cada una se colocaron $5 \mathrm{~g}$ de materia seca de cada tratamiento o forraje por duplicado en diferentes horas $(6,12,24,48$ y 72), con el fin de establecer una curva de degradabilidad de la materia seca, fibra detergente neutro, fibra detergente ácido y nitrógeno total. En el líquido ruminal se realizaron mediciones de $\mathrm{pH}$ y nitrógeno amoniacal a las $0,4,8$ y 12 horas. Los forrajes secos de matarratón, morera y nacedero fueron sometidos a un análisis nutricional preliminar, en el cual se le determinó a cada uno materia seca (MS), extracto etéreo (EE), nitrógeno total (NT), cenizas, fibra cruda (FC), fibra detergente neutro (FDN), fibra detergente ácido (FDA) y extracto no nitrogenado (ENN), cuatro repeticiones por forraje con el fin de conocer parcialmente la caracterización bromatológica que posee cada tratamiento, lo cual se realizó antes de medir su degradabilidad en el rumen siguiendo la metodología de la AOAC, (2006). Se calculó la tasa de degradación mediante la fórmula de Méhrez y Orskov, (1977) para tal afecto:

$$
\gamma=\alpha+\beta\left(1-e^{c(\tau-l)}\right)
$$

El modelo estadístico fue un cuadrado latino $3 \times 3$, utilizando tres animales con tres tratamientos. Las variables analizadas fueron la degradación ruminal y su tasa en diferentes horas $(6,12,24,48$ y 72$)$ de la MS, FDN, FDA y NT de los tres tratamientos. En el líquido ruminal se evaluó el pH y el nitrógeno amoniacal a las 
0, 4, 8 y 12 horas. Se utilizó un análisis de varianza, procedimiento GLM (SAS) aplicando la prueba de Duncan para comparación de medidas. Este modelo estadístico es el más utilizado cuando se trabaja con animales fistulados en rumen debido a que se obtienen suficientes repeticiones de las muestras analizadas en poca cantidad de animales, siendo confiables desde el punto de vista numérico y estadístico.

\section{RESULTADOS Y DISCUSIÓN}

Los resultados del análisis bromatológico del matarratón (Tabla 1) en lo que se refiere a proteína y fibra detergente neutro concuerdan con los encontrados por Benavides, (1995) siendo estos de $24.8 \%$ y $45 \%$ respectivamente, igual sucedió con la proteína y la FDN de la morera: en la primera reportó Kundu y Sharma, (1988) un $19.6 \%$, dato cercano al obtenido en este trabajo que fue de $18.6 \%$, y en la segunda, Flores et al., (1998) encontraron que ésta era de $29.8 \%$ valor similar al de este trabajo (Tabla 1), mientras que Benavides, (1995) y Flores et al., (1998) hallaron que el contenido de este nutriente fue de $24 \%$, esta diferencia se debe posiblemente a la influencia que tiene la edad de la planta, la posición de la hojas y nivel de fertilización que pueden dar una variabilidad a los valores analizados en el laboratorio. En la composición nutricional del nacedero (Tabla 1) se observa que la proteína (19.2\%) fue similar a la hallada por Gómez et al., (1995) (18\%) y Flores et al., (1998) (19.9\%), también los datos de FDN concuerdan con los obtenidos por estos últimos autores en 1998 (40.7\%).

La morera presentó las mayores tasas de degradación en cuanto a MS (Figura 1), $\mathrm{NT}$, FDA y FDN $(\mathrm{P}<0.05)$, alcanzando esta última la mayor degradabilidad a las 72 horas (81.9\%), aunque su tasa de degradación fue la más lenta en un principio comparándola con la del matarratón (Tabla 2 y Figura 2). El matarratón obtuvo una tasa de degradación rápida de la FDN (9.4\% a las 12 horas) (Tabla 2), sin embargo no se mantiene igual hasta las 72 horas ya que se estabiliza en valores cercanos al $64.1 \%$ (Figura 2) siendo la degradación de la FDN más baja de las tres especies estudiadas $(P<0.05)$. El nacedero presenta la tasa de degradación más baja en lo que corresponde a la MS, FDN, FDA y NT $(\mathrm{P}<0.05)$ (Tabla 2), este 
último nutriente tiene una degradación lenta en un principio, lo que puede estar influenciado por la dificultad en la liberación de nitrógeno por la lenta degradación de la pared celular, sin embargo la degradabilidad del NT a las 72 horas es mayor que la del matarratón (Figura 3). Los resultados de la tasa de degradación de la materia seca en el nacedero y la morera son menores a los reportados por Flores et al., (1998) (6.3 y $3.32 \%$ de material degradado/hora).

Tabla 1. Análisis nutricional del matarratón (Gliricidia sepium), morera (Morus alba) y nacedero (Trichanthera gigantea), en el presente estudio

\begin{tabular}{cccc}
\hline ESPECIE & Gliricidia sepium & Morus alba & $\begin{array}{c}\text { Trichanthera } \\
\text { gigantea }\end{array}$ \\
\hline VARIABLE (\%) & 26.8 & 34.0 & 20.2 \\
Materia seca & 22.1 & 18.6 & 19.2 \\
Proteína total & 12.4 & 8.9 & 2.8 \\
Fibra cruda & 49.0 & 28.3 & 42.8 \\
FDN & 21.1 & 17.4 & 27.0 \\
FDA & 3.4 & 2.5 & 4.3 \\
Extracto Etéreo & 48.7 & 54.6 & 50.1 \\
ENN & 8.2 & 11.1 & 16.6 \\
Cenizas & & &
\end{tabular}

Aunque el matarratón presentó inicialmente un $48.1 \%$ de degradabilidad de la MS a las 6 horas (Figura 1), a las 72 horas fue la menor $(P<0.05)$ en comparación con las otras dos especies que obtuvieron valores de 91 y $85.3 \%$ para morera y nacedero respectivamente, además se observó que los datos de degradación de la materia seca del matarratón a las 24 y 48 horas (Figura 1) son ligeramente superiores a los reportados por Benavides en 1995 (60\%), la morera también mostró degradabilidades a estas mismas horas más elevadas (81.6 y $88.3 \%$ ) con relación a las encontradas por Kundu y Sharma en 1988 (75 y 85\%), el comportamiento de esta variable en las tres especies a través del tiempo se observa en la Figura 1. 


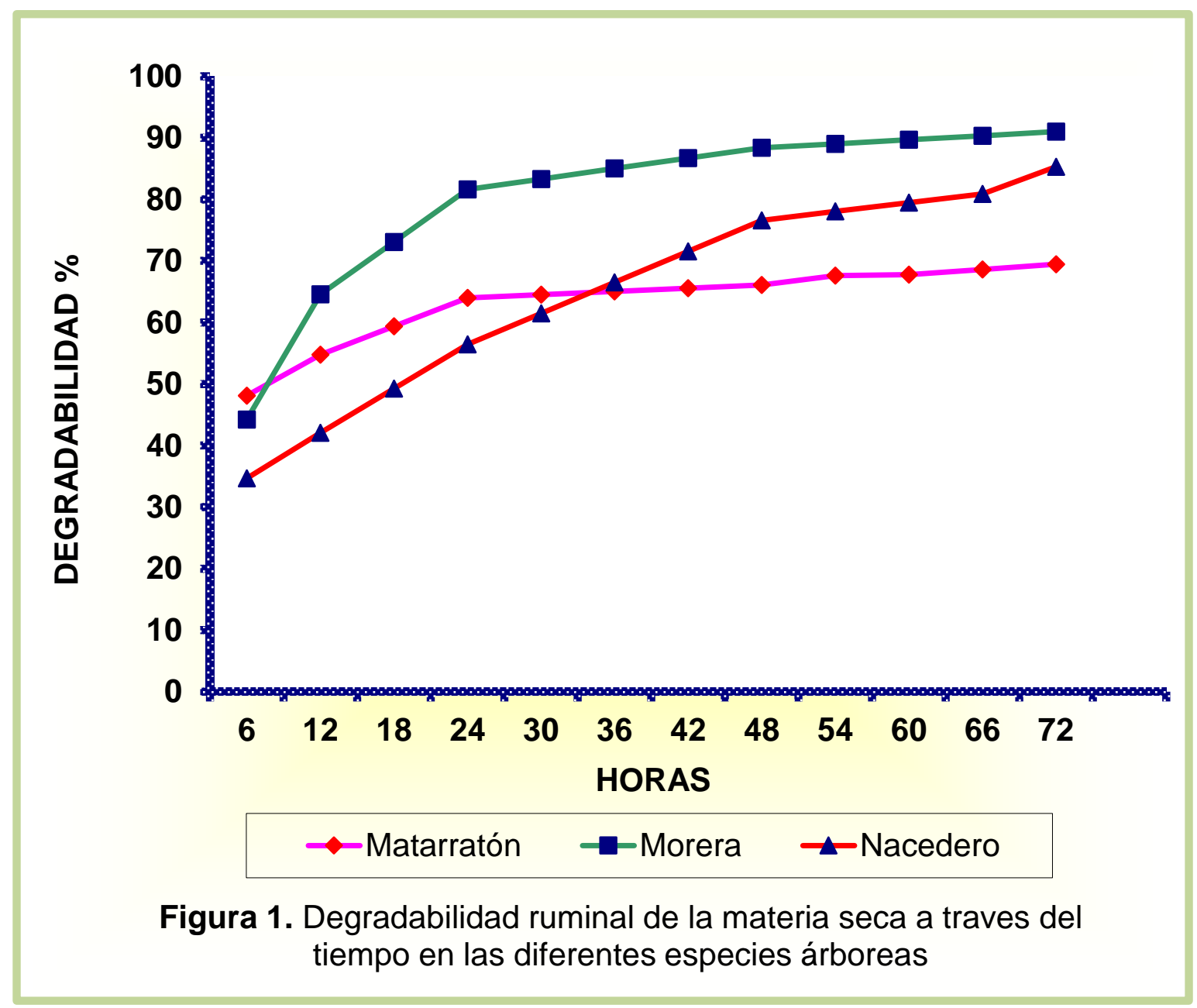

Tabla 2. Tasa de degradación (\% de material/hora) de las tres especies arbóreas estudiadas

\begin{tabular}{cccc}
\hline PARAMETRO & $\begin{array}{c}\text { Gliricidia } \\
\text { sepium }\end{array}$ & Morus alba & $\begin{array}{c}\text { Trichanthera } \\
\text { gigantea }\end{array}$ \\
\hline "C" Materia seca & $6.5^{\mathrm{b}}$ & $7.7^{\mathrm{a}}$ & $3.8^{\mathrm{c}}$ \\
"C" Nitrógeno total & $5.7^{\mathrm{b}}$ & $7.5^{\mathrm{a}}$ & $0.1^{\mathrm{c}}$ \\
"C" Fibra en detergente neutro & $9.4^{\mathrm{a}}$ & $3.7^{\mathrm{b}}$ & $3.2^{\mathrm{c}}$ \\
"C" Fibra en detergente ácido & $3.3^{\mathrm{b}}$ & $10.0^{\mathrm{a}}$ & $3.2^{\mathrm{c}}$ \\
\hline
\end{tabular}

Letras distintas en la misma fila son diferentes $(\mathrm{P}<0.05)$ 


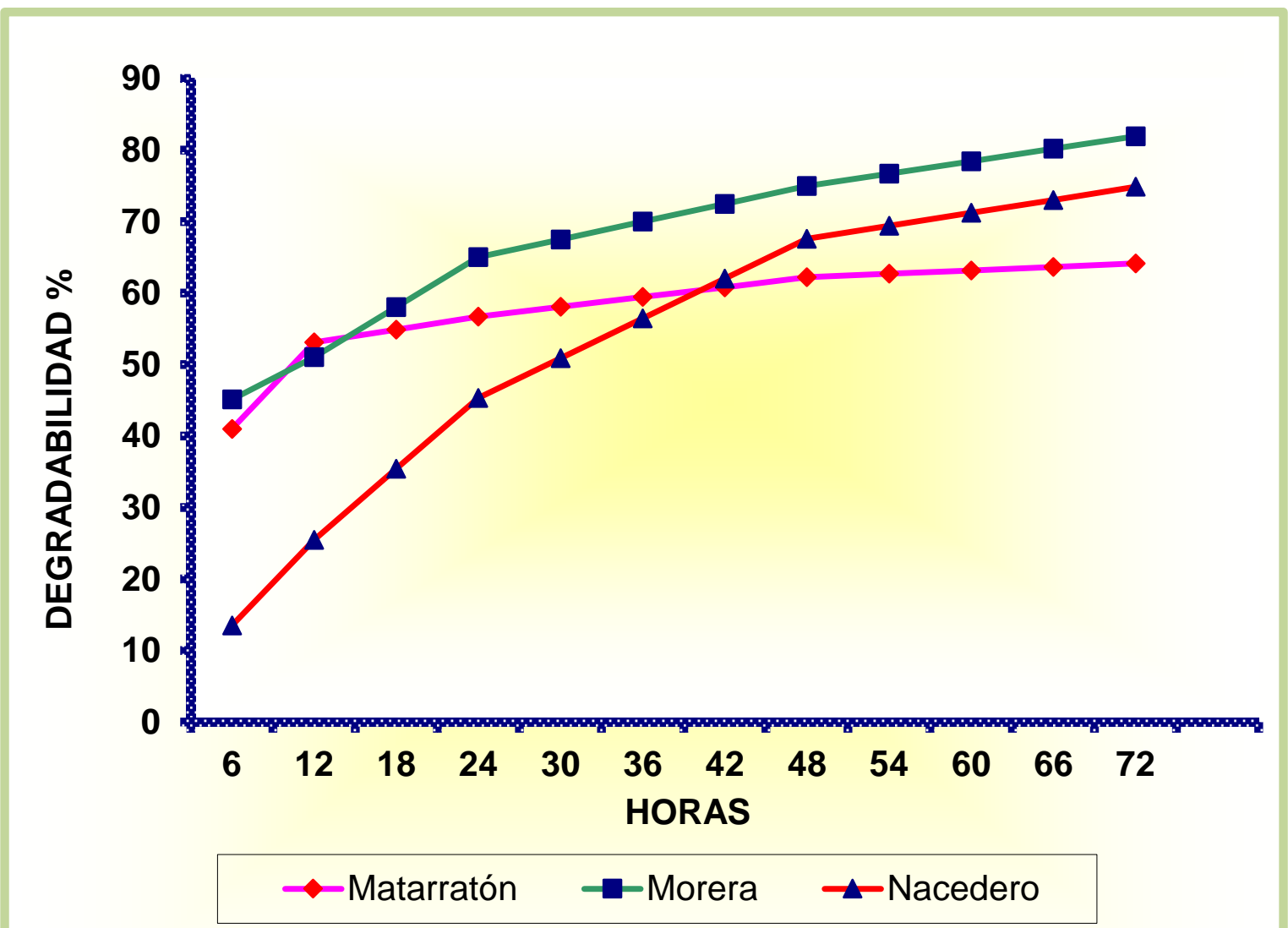

Figura 2. Degradabilidad ruminal de la fibra en detergente neutro a través del tiempo en las tres especies arbóreas

En la degradabilidad de la fibra detergente neutro, el análisis de varianza muestra que el nacedero tiene inicialmente (6 y 12 horas) la menor degradación $(P<0.05)$ en comparación con las otras dos especies (Figura 2). A las 72 horas el matarratón presentó la más baja degradación de la FDN $(\mathrm{P}<0.05)$ con relación a la morera y nacedero (Figura 2), lo que indica una mayor presencia de componentes solubles en la pared celular de estas dos últimas especies lo cual se demuestra en su menor contenido de fibra detergente neutro, 21.3 y $42.8 \%$ respectivamente en comparación con la del matarratón (49\%). La degradación de la fibra detergente ácido a las 72 horas fue menor $(\mathrm{P}<0.05)$ para el matarratón $(46.7 \%)$ con respecto a la morera y nacedero que obtuvieron valores de 76.9 y $73.8 \%$ respectivamente (Figura 3), este nutriente mostró un comportamiento ruminal similar al de la FDN en las diferentes horas de incubación. 


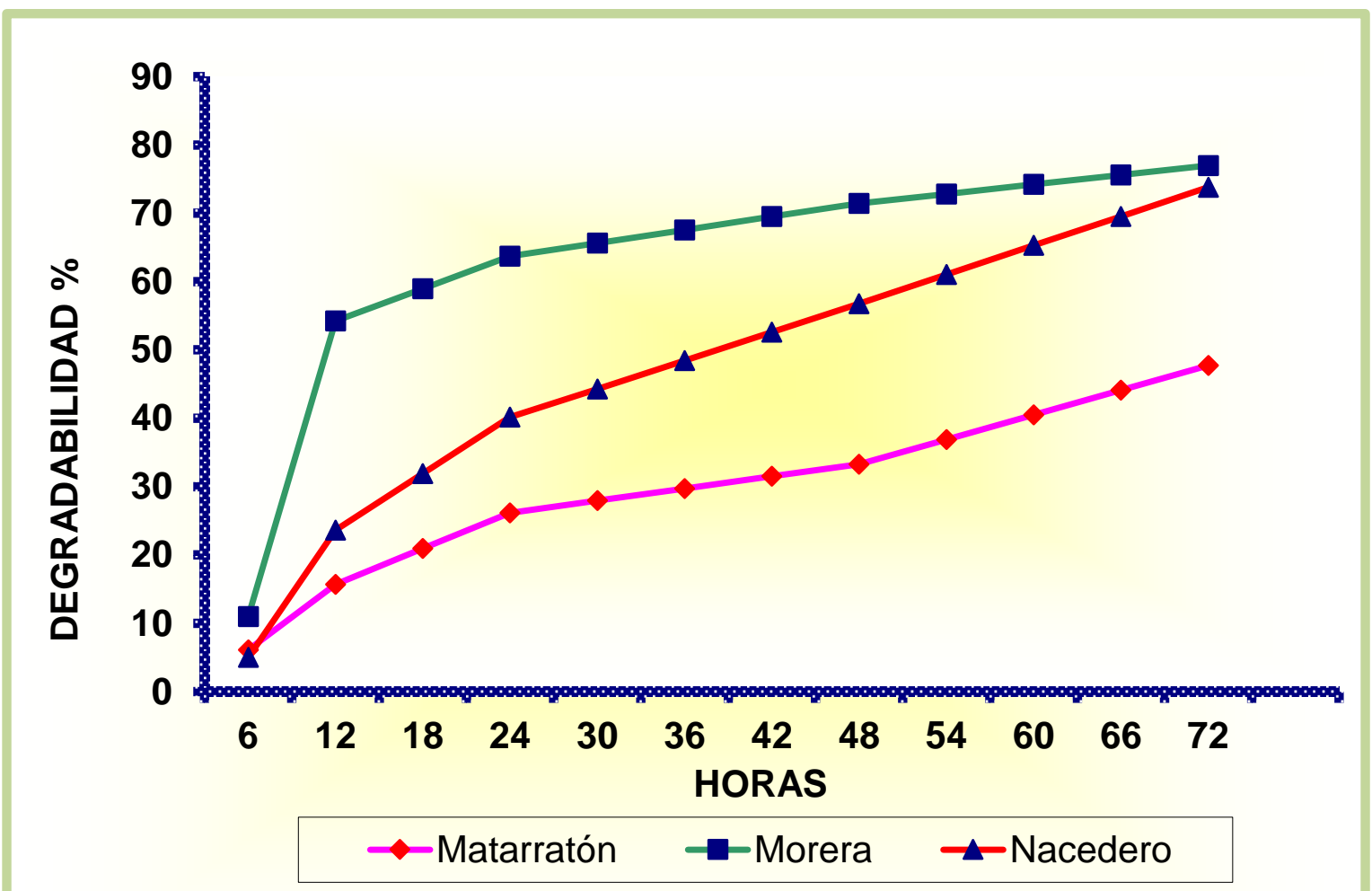

Figura 3. Degradabilidad ruminal de la fibra en detergente ácido a través del tiempo de las tres especies arbóreas

La morera mostró la degradabilidad de nitrógeno total más elevada en todas las horas $(P<0.05)$, aunque el matarratón presentó una mayor degradabilidad hasta las 24 horas $(P<0.05)$ en comparación con el nacedero, a las 48 y 72 la de este último fue mayor numéricamente, lo cual se debió a que la FDN incrementó su degradación después de las 24 horas, mientras que el matarratón degradó más del $80 \%$ de la FDN a las 12 , dejando más cantidad de nitrógeno disponible para las bacterias en las primeras horas (Figura 4). En los parámetros evaluados en el líquido ruminal de nitrógeno amoniacal y $\mathrm{pH}$ no se observaron diferencias en casi la totalidad de las horas excepto en nacedero que presentó un pH de 4.7 a las 12 horas siendo más bajo $(\mathrm{P}<0.05)$ en comparación con las otras dos especies (Tabla $3)$. 
Tabla 3. Nitrógeno amoniacal (mg/100ml de líquido ruminal) y pH en líquido ruminal

\begin{tabular}{ccccc}
\hline & Horas & $\mathbf{0}$ & $\mathbf{8}$ & $\mathbf{1 2}$ \\
\hline Especie & \multicolumn{5}{c}{ Nitrógeno amoniacal } \\
Matarratón & $27.4 \mathrm{a}$ & $28.1 \mathrm{a}$ & $15.9^{\mathrm{a}}$ & $18.7 \mathrm{a}$ \\
Morera & $38.2 \mathrm{a}$ & $40.1 \mathrm{a}$ & $29.3^{\mathrm{a}}$ & $25.7 \mathrm{a}$ \\
Nacedero & $35.4 \mathrm{a}$ & $22.2 \mathrm{a}$ & $16.4^{\mathrm{a}}$ & $30.3 \mathrm{a}$ \\
& & $\mathrm{pH}$ & & \\
Matarratón & $6.4 \mathrm{a}$ & $6.0 \mathrm{a}$ & $5.8^{\mathrm{a}}$ & $5.6 \mathrm{a}$ \\
Morera & $6.5 \mathrm{a}$ & $5.8 \mathrm{a}$ & $5.6^{\mathrm{a}}$ & $5.5 \mathrm{a}$ \\
Nacedero & $6.3 \mathrm{a}$ & $5.8 \mathrm{a}$ & $5.7^{\mathrm{a}}$ & $4.7 \mathrm{~b}$ \\
\hline
\end{tabular}

Letras distintas en la misma columna son diferentes $(P<0.05)$

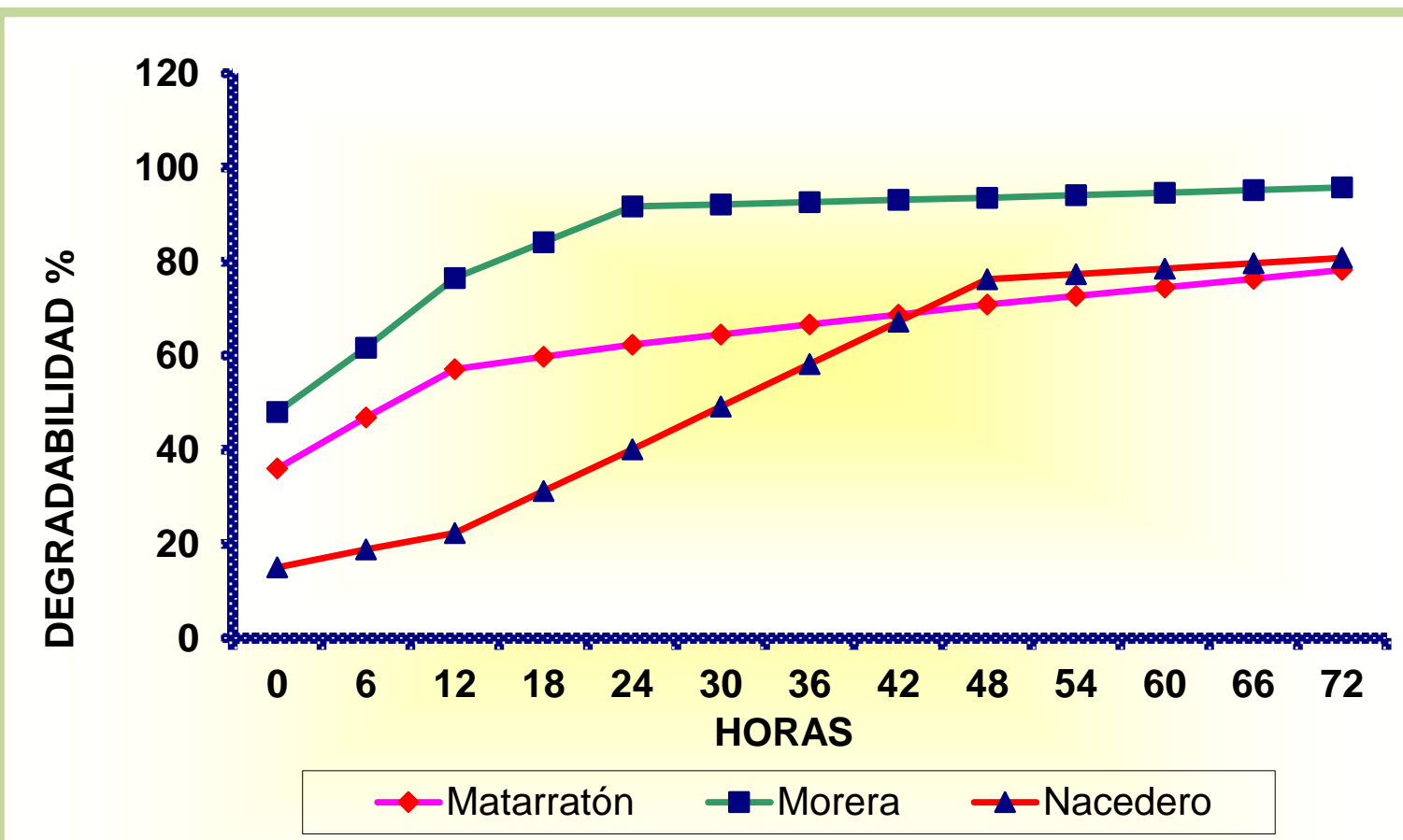

Figura 4. Degradabilidad ruminal del Nitrógeno total a través del tiempo de las tres especies arbóreas 


\section{CONCLUSIONES}

La mayor degradabilidad ruminal a las 72 horas de la materia seca, fibra detergente neutro, fibra detergente ácido y nitrógeno total la presentó la morera (Morus alba) en comparación con nacedero (Trichanthera gigantea) y matarratón (Gliricidia sepium).

También se observaron diferencias en las tasas de degradabilidad ruminal de los nutrientes evaluados en la morera (Morus alba), nacedero (Trichanthera gigantea) y matarratón (Gliricidia sepium), siendo mayores en la morera y menores en el nacedero.

La suplementación con las tres especies estudiadas ocasiona un descenso en el $\mathrm{pH}$ del líquido ruminal y no se encuentran diferencias entre los efectos de las especies. El nitrógeno amoniacal no se mantuvo constante en las horas evaluadas, presentándose en la morera y el matarratón una mayor concentración a las cuatro horas y en el nacedero a las cero horas, tampoco se observaron diferencias estadísticas entre las tres especies.

\section{RECOMENDACIONES}

Se recomienda que estudios posteriores se encaminen a determinar las cantidades que se deben utilizar de estas tres especies forrajeras en dietas para rumiantes para así comprender mejor los procesos que se suceden en el interior del rumen, y su efecto que tienen sobre la producción.

\section{REFERENCIAS BIBLIOGRÁFICAS}

1. Association of Official Analytical Chemists (AOAC). 2006. Official Methods of Analysis. $18^{\text {th }}$ Ed., Washington, D. C.

2. Benavides J. Manejo y utilización de la morera como follaje. Agroforestería de las Américas, 2 (7): 27-30. 1995.

3. Benavides JE. Árboles y arbustos forrajeros en América Central. Turrialba. Costa Rica. CATIE. 2: 423-721. 1995.

4. Elizondo SJ. Calidad nutricional y consumo de morera (Morus alba), ramio (Bohemeria nivea) y sorgo negro forrajero (Sorghum almum) en cabras. Agronomía Mesoamericana, 15 (2): 209 -213. 2004. 
5. Flores OI, Bolívar DM, Botero JÁ, Ibrahim MA. Parámetros nutricionales de algunas arbóreas leguminosas y no leguminosas con potencial forrajera para la suplementación de rumiantes en el trópico. Livestoack Research for Rural Development, 10 (1): 10-15. 1998.

6. Frioni L. Procesos Microbianos. Ed Fundación Universidad Nacional de Rio Cuarto Argentina. Rio Cuarto Argentina: 330 p. 1999.

7. Gómez ME, Rodríguez L, Murgueitio E, Ríos C, Molina C, Molina H, Molina E, Molina P. Matarratón (Gliricidia sepium) y nacedero (Trichanthera gigantea). En árboles y arbustos forrajeros utilizados en alimentación animal como fuente proteica. CIPAV, Cali, Colombia 129 p. 1995.

8. Hurtado DI, Nocua S, Nárvaez W, Vargas J. Valor nutricional de la morera (Morus sp.), matarratón (Gliricidia sepium), pasto india (Panicum maximun) y arboloco (Montanoa quadrangularis) en la alimentación de cuyes (Cavia porcellus). Revista Veterinaria Zootecnia, 6 (1): 56-65. 2012.

9. Kundu S, Sharma V. Chemical composition and in vitro dry matter digestibility of certain tree leaves. Journal of Dairy Science 72 (12): 3233-3239. 1988.

10. Mehrez AL, Orskov ER. A Study of artificial bag technique for determining the digestibility of feed in the rumen. Journal of Agriculture Science, 88: 645-650. 1977.

11. Nieves D, Araque H, Terán O, Silva L, González C, Uzcátegui W. Digestibilidad de nutrientes del follaje de Morera (Morus alba) en conejos de engorde. Revista Científica Maracaibo, 16 (4): 315-324. 2006.

12. Orskov ER. Nutrición proteica de los rumiantes. Ed. Actribia. Zaragoza. 160 p. 1988.

13. Owens FN, Goetsch LA. Ruminal fermentation. In Church. Rumiant animal, digestive physiology and nutrition. Prentice Hall New Jersey, p 195-225. 1988.

14. Roa ML. Efecto de Sachaaromyces cerevisae y tres tipos de fibra en la degradabilidad ruminal de nutrientes en bovinos. Tesis MSc. Colegio de Postgraduados, Chapingo México. 105 p. 1992.

15. Rodríguez J., Elizondo JA. Consumo, calidad nutricional y digestibilidad aparente de morera (Morus alba) y pasto estrella (Cynodon nlemfuensis) en cabras. Revistas Agronomía Costarricense, 36 (1): 13-23. 2012.

16. Suárez JC, Carulla JE, Velásquez JE. Composición química y digestibilidad in vitro de algunas especies arbóreas establecidas en el piedemonte Amazónico. Zootecnia Tropical, 26 (3): 231-234. 2008.

17. Wattiux MA, Howard RT. Digestión en la vaca lechera. 2000. Recuperado 5 de Febrero 2013. Disponible En: www.babcock.cals.wisc.edu/bab/des/digest/ch1/dig.html

18. Wilkings RJ. The potential digestibility of cellulose in forage and feces. Journal of Agriculture Science, (3): 57-60. 1990. 